\title{
Effect of Glow Discharge Treatment of Poly(Acrylic Acid) Preadsorbed onto Poly (ethylene)
}

\author{
JOHANNES G. A. TERLINGEN, ${ }^{1}$ ALLAN S. HOFFMAN, ${ }^{2}$ and JAN FEIJEN ${ }^{1, *}$ \\ ${ }^{1}$ Department of Chemical Technology, University of Twente, P.O. Box 217, 7500 AE Enschede, The Netherlands and \\ ${ }^{2} \mathrm{Ctr}$. for Bioengineering $\mathrm{Fl}-20$, University of Washington, Seattle, Washington 98195
}

\begin{abstract}
SYNOPSIS
In order to introduce carboxylic acid groups at the surface of poly (ethylene) (PE) films, an attempt was made to covalently link a preadsorbed layer of poly (acrylic acid) (PAAc) on a $\mathrm{PE}$ film by an argon or tetrafluoromethane $\left(\mathrm{CF}_{4}\right)$ plasma treatment. Surface analysis was performed by XPS (X-ray photoelectron spectroscopy) and water contact angle measurements. It was shown that by treatment of a PAAc layer preadsorbed on PE with an argon or a $\mathrm{CF}_{4}$ plasma, a small amount of carboxylic acid groups was introduced at the surface. A similar amount of these groups was obtained by plasma treatment of PE films without a preadsorbed PAAc layer. A comparison of the etching rates of PAAc and PE by either an argon or a $\mathrm{CF}_{4}$ plasma, showed that PAAc is etched much faster by both types of plasmas than PE. The preadsorbed PAAc layer on PE is etched off before it could be immobilized by either an argon or a $\mathrm{CF}_{4}$ plasma treatment. Additionally the effect of treating $\mathrm{PE}$ films for very short times with an argon or $\mathrm{a} \mathrm{CF}_{4}$ plasma was studied. After an induction period of approximately $0.1 \mathrm{~s}$, the oxidation during the argon plasma treatment and the fluorination during the $\mathrm{CF}_{4}$ plasma treatment were proportional to the logarithm of the treatment time for time periods up to 50 s. (C) 1993 John Wiley \& Sons, Inc.
\end{abstract}

\section{INTRODUCTION}

During the last decade, functionalization of polymer surfaces has evolved to a very prominent field of research. The presence of functional groups at the surfaces is important for processes like adhesion and wettability. Furthermore they can be used for the covalent binding of bioactive molecules for biotechnological and biomedical applications. Because carboxylic acid groups are particularly suitable for the coupling of amine or hydroxyl containing bioactive molecules to solid surfaces, ${ }^{1}$ a considerable effort has been made to introduce these groups at hydrophobic polymer surfaces. Generally two types of functionalization processes can be distinguished: namely, wet and dry chemistry approaches. By using solutions of strong acids and oxidizing agents it is possible to oxidize poly(ethylene) (PE) and poly (propylene) (PP) surfaces to such a degree that

\footnotetext{
* To whom correspondence should be addressed. Journal of Applied Polymer Science, Vol. 50, 1529-1539 (1993) (C) 1993 John Wiley \& Sons, Inc. CCC 0021-8995/93/091529-11
}

carboxylic acid groups are introduced at the surface. ${ }^{2-5}$ Many different graft processes have also been reported in which a monomer, for example, acrylic acid, is grafted onto a polymer substrate by means of gamma irradiation, ${ }^{6-8}$ fast electrons, ${ }^{9,10}$ or UV. ${ }^{11}$ The main disadvantage of wet chemistry approaches is that the modification is not always restricted to the outermost surface. ${ }^{12}$

With many of the dry chemistry functionalization techniques, such as corona and plasma treatments, it is possible to selectively modify the outermost surface. Plasma techniques have been widely used to introduce functional groups on polymer surfaces. ${ }^{13}$ For instance plasma polymerization of acrylic acid $^{14,15}$ or of acrylic acid/carbon dioxide ${ }^{16}$ mixtures yields carboxylic acid groups on the surface. Different plasma-induced grafting processes, for instance grafting of acrylic acid on poly (ethylene terephtalate $)^{17}$ and $\mathrm{PE}^{18,19}$ surfaces, have been applied for the functionalization of polymer surfaces. Furthermore by applying an oxidizing plasma, different oxygen-containing functional groups are introduced at the polymeric surface. ${ }^{20}$ 
A limiting factor for the widespread use of plasma processes is the complexity of the chemistry of the plasma phase. As a consequence the chemical composition of the surface obtained by plasma processes is usually less defined than desired. To circumvent this problem we have developed a new approach that involves plasma immobilization of preadsorbed surface-active compounds on inert polymer substrates. In this process a compound with the desired functionality is preadsorbed on a polymer surface and then plasma treated with an inert gas to induce a covalent coupling of the preadsorbed compound to the surface. By immobilizing a preadsorbed sodium dodecylsulfate (SDS) layer on PP it is possible to introduce sulfate groups at the surface.$^{21}$ Furthermore by immobilization of decylamine hydrochloride on PE, amine groups are introduced at the PE surface. ${ }^{22}$ Finally fibrinogen-repellant "nonfouling" surfaces are obtained by the immobilization of a poly (ethylene oxide) (PEO) containing surfactant on $\mathrm{PE}{ }^{23,24}$

In this study the applicability of the plasma immobilization method for the introduction of carboxylic acid groups onto a solid hydrophobic polymer surface has been investigated. A schematic presentation of the plasma immobilization process is given in Figure 1. In the first step a surface active carboxylic acid containing compound is adsorbed on a hydrophobic surface. Rothman ${ }^{25}$ has reported that poly (acrylic acid) (PAAc) adsorbs reversibly onto a hydrophobic gel, and based on this report it was assumed that this adsorption behavior could be extrapolated to other hydrophobic surfaces. Therefore the adsorption and desorption behavior of PAAc on PE was studied first. Subsequently PE films with a preadsorbed PAAc layer (PE/PAAc films) were treated with an argon or a tetrafluoromethane $\left(\mathrm{CF}_{4}\right)$ plasma to investigate whether this layer could be immobilized onto the PE film with retention of the carboxylic acid groups. After washing to remove any unbound PAAc from the plasma-treated surface,

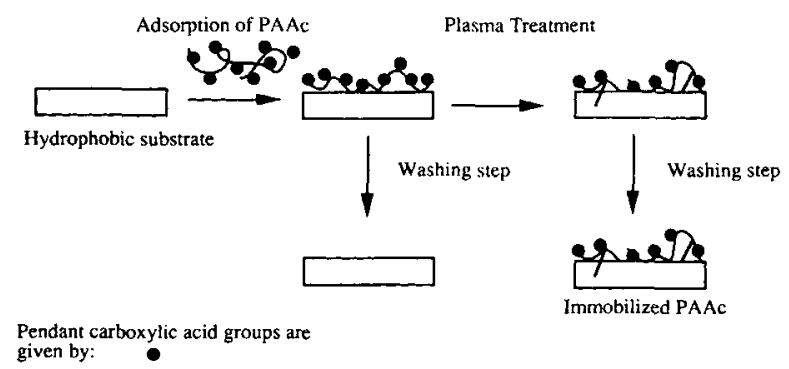

Figure 1 Schematic presentation of the plasma immobilization of a preadsorbed layer of PAAc on PE. surfaces were analyzed with X-ray photoelectron spectroscopy (XPS) and water contact angle measurements. Furthermore specific reactions for carboxylic acid groups followed by XPS measurements ${ }^{26,27}$ were utilized to quantify the surface concentration of carboxylic acid groups.

\section{EXPERIMENTAL}

\section{Materials}

PE film (no additives) was low density, type 2300 , DSM, Geleen, The Netherlands. PAAc was purchased from Aldrich Chemie, Brussels, Belgium. Two batches with different molecular weights were used. PAAc with a molecular weight $\left(M_{w}\right)$ of 5,000 $\mathrm{g} / \mathrm{mol}$ (delivered as a $50 \% \mathrm{w} / \mathrm{v}$ solution in water) was used for the adsorption experiments. For the preparation of pure PAAc films a $M_{w}$ of $250,000 \mathrm{~g} /$ mol was used. Poly (ethylene-co-acrylic acid) [P(EcoAAc), random copolymer $(92: 8)]$ was obtained from Polysciences Inc., Warrington, USA. Dichloromethane (GR), toluene (GR), acetone (GR), methanol (GR), pyridine (GR), sodium chloride ( $\mathrm{NaCl}, \mathrm{GR}$ ), SDS (purity $>99 \%$ ), trifluoroethanol (TFE, purity $>99 \%$ ), aqueous sodium hydroxide $(1.00 \mathrm{M} \mathrm{NaOH})$, and aqueous hydrochloric acid $(1.00 \mathrm{M} \mathrm{HCl})$ were purchased from Merck, Darmstadt, Germany. The GR grade chemicals have a purity of at least $99.5 \%$. Chlorotrimethylsilane (purity $>99 \%$ ) was obtained from Fluka AG, Buchs, Switzerland and 1,3-di-tert-butylcarbodiimide (purity 99\%) was obtained from Aldrich Chemie. Argon ( $\mathrm{Ar}$, purity $\geq 99.999 \%$ ) and $\mathrm{CF}_{4}$ (purity $\geq 99.95 \%$ ) were purchased from Hoekloos, Amsterdam, The Netherlands. For all experiments doubly deionized water was used.

\section{Methods}

Films were routinely stored at room temperature (RT) under atmospheric pressure in glass vials in the dark.

\section{Preparation of Polymer Films}

Preparation of PAAc Films. PAAc $(10 \mathrm{~g})$ was dissolved in $500 \mathrm{~mL}$ of methanol. The solution was cast onto two clean petri dishes (diameter $19 \mathrm{~cm}$ ). After evaporation of the methanol at RT, the PAAc films were dried in vacuo at RT.

Preparation of $P(E c o A A c)$ Films. P(EcoAAc) films were prepared in a silanized petri dish because of the very strong adhesion forces between clean glass 
and the films of the copolymer. Petri dishes were silanized by a treatment with a solution of chlorotrimethylsilane in toluene $(30 \% \mathrm{v} / \mathrm{v})$ for $10 \mathrm{~min}$, followed by rinsing five times with toluene and rinsing three times with methanol. Subsequently $\mathrm{P}(\mathrm{EcoAAc})(5 \mathrm{~g})$ was dissolved in $30 \mathrm{~mL}$ of toluene at $80^{\circ} \mathrm{C}$ in a vacuum stove. The toluene was slowly stripped off, and the films were dried for several hours at $80^{\circ} \mathrm{C}$ in vacuo.

Cleaning of PE Films. PE films $(13 \times 25 \mathrm{~mm})$ were cleaned ultrasonically in dichloromethane for 10 min. Fresh dichloromethane was added and the procedure was repeated. The total cleaning procedure consisted of treatment four times with dichloromethane, four times with acetone, and four times with water. Subsequently the films were dried in vacuo and stored.

\section{Adsorption and Desorption of PAAc on PE}

Adsorption of PAAc on PE. Each PE film was placed in $5 \mathrm{~mL}$ of an aqueous solution of PAAc $(1 \% \mathrm{w} / \mathrm{v}$, $\mathrm{pH} 3.2$ ) at RT. After $1 \mathrm{~h}$ the film was taken out, rinsed (once) with water, and dried in vacuo. These films are denoted as PE/PAAc films.

Desorption (Washing) of Adsorbed PAAc. PE/ PAAc films were washed with aqueous solutions of either $\mathrm{NaCl}(3.0 M), \mathrm{NaOH}(1.00 M), \mathrm{HCl}(1.00 M)$, or $\operatorname{SDS}(1 \% \mathrm{w} / \mathrm{v})$ for $1 \mathrm{~h}$ at RT. After removal of these solutions, the films were washed three times with water and dried in vacuo.

\section{Plasma Treatment}

A schematic presentation of the plasma treatment system used is given in Figure 2.

Plasma Treatment of PE/PAAc and PE Films. PE/ PAAc (six films) and PE films (six films) were placed on a glass plate in the center region of the reactor and the reactor was evacuated (pressure $p$ $<1 \cdot 10^{-5} \mathrm{mbar}$ ). Subsequently a gas flow (Ar or $\mathrm{CF}_{4}$ ) of $10 \mathrm{~cm}^{3}$ (STP) $/ \mathrm{min}$ was established through the reactor. After $15 \mathrm{~min}$ the films were plasma treated ( $44 \mathrm{~W}, 0.07$ mbar) for time periods ranging from 0.05 to $50 \mathrm{~s}$. Two minutes after the plasma treatment, the reactor was brought to atmospheric pressure with air. The other side of the films was treated according to the same procedure.

Washing of Plasma-Treated Films. Plasma-treated $\mathrm{PE}$ and PE/PAAc films were immersed in an aqueous $\mathrm{NaOH}$ solution (1.00M) at RT. After $1 \mathrm{~h}$

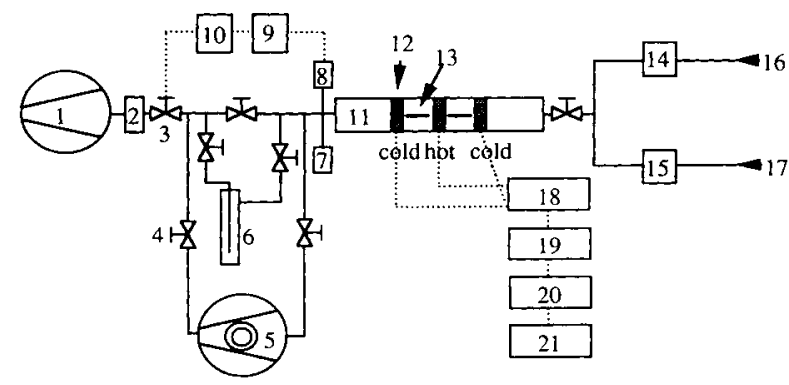

Figure 2 Schematic presentation of the plasma treatment system used. The system consists of the following components. 1: Two stage rotary pump (DUO 030 A EX, Balzers BV, Utrecht, The Netherlands ); 2: zeolite trap to prevent oil back-streaming (Balzers); 3: throttle valve (type $253 \mathrm{~A}$, MKS Instruments, München, Germany) directly connected to the pressure gauge to have an independent control of the process pressure; 4: open/close vacuum valves; 5 : turbomolecular pump (TPH 060, Balzers); 6: cold trap; 7: cold cathode pressure head (IKR $020+$ TPF 300 control unit, Balzers); 8: pressure gauge [Baratron (type 122A), MKS]; 9: control unit Baratron (PR2000, MKS); 10: control unit throttle valve (Type 252A, MKS); 11: reactor, dimensions: length, $80 \mathrm{~cm}$, internal diameter, $6.5 \mathrm{~cm}$; 12 : three externally placed capacitively coupled RF electrodes, spaced at $10-\mathrm{cm}$ intervals; 13: polymer samples on glass plates; 14, 15: mass flow controllers (Type 1259 B + PR3000 control unit, MKS); 16, 17: gas inlets; 18: matching network (ENI Matchwork 5, ENI Power Systems, Rochester, NY); 19: control unit matching network (ENI TH-1000, ENI); 20: RF (13.56 $\mathrm{MHz}$ ) generator (ENI ACG-3, ENI); 21: function generator (Wavetek $5 \mathrm{MHz}$ XCG/sweep generator model 183, Wavetek San Diego, CA), used as a remote control for the generator to enable very short plasma treatment times $(t \geq 0.01 \mathrm{~s})$.

the films were rinsed twice with methanol and dried in vacuo. Films to be used for contact angle measurements were washed three times with water instead of methanol.

Determination of Etching Rates of PE and PAAc Films. Two films (PE or PAAc, $4 \times 10 \mathrm{~cm}$ each) were placed on a glass plate in the center region of the reactor and the reactor was evacuated ( $p$ $<5 \cdot 10^{-6}$ mbar). Subsequently the films were taken out, immediately weighed, and returned to the reactor. If no weight decrease of the films was measured, the following plasma treatment procedure was applied. After placing the films in the reactor, the reactor was evacuated $\left(p<1.10^{-5} \mathrm{mbar}\right)$. A gas flow (Ar, $\mathrm{CF}_{4}$, or air) of $10 \mathrm{~cm}^{3} / \mathrm{min}$ was established through the reactor. After $15 \mathrm{~min}$ the films were plasma treated ( $44 \mathrm{~W}, 0.06-0.07$ mbar) for $3 \mathrm{~h}$. Two minutes after the plasma treatment, the reactor was 
brought to atmospheric pressure with air. The films were taken out and immediately weighed. The gravimetrically determined weight decrease was converted into an average etching rate.

\section{Characterization}

Contact Angle Measurements. Wilhelmy plate, water contact angle measurements ${ }^{28,29}$ were performed with all modified and control surfaces at RT.

XPS Measurements. XPS measurements were performed with a Kratos XSAM-800 (Manchester, United Kingdom) apparatus using a $\mathrm{Mg} \mathrm{K}_{\alpha}$ source (15 kV, $15 \mathrm{~mA}$ ). The samples were analyzed with the analyzer perpendicular to the sample surface. A spot size with a diameter of $3 \mathrm{~mm}$ was analyzed. The pressure during measurements was typically $1 \cdot 10^{-8}$ mbar. The spectra were recorded in the low resolution mode (pass energy $40 \mathrm{eV}$, FWHM Ag3d ${ }^{5 / 2}$ : $1.2 \mathrm{eV}$ ) and standard sensitivity factors (delivered by Kratos) were used to convert the measured peak areas into atom percentages. Whenever fluorinecontaining surfaces were analyzed, a clean PTFE sample was also analyzed to determine a correct empirical sensitivity factor for the fluorine 1 s peak.

Scanning Electron Microscopy (SEM). Modified and control films were characterized using a JEOL type JSM-35CF apparatus. The films were sputtered with gold (with a Balzers Union Sputter apparatus), and analyzed at an angle of $60^{\circ}$ at different magnifications.

\section{Derivatization of Carboxylic Acid Groups for XPS Analysis}

The reactions used to tag carboxylic acid groups on surfaces in order to be able to detect and quantify these groups with XPS, are given in Scheme 1.

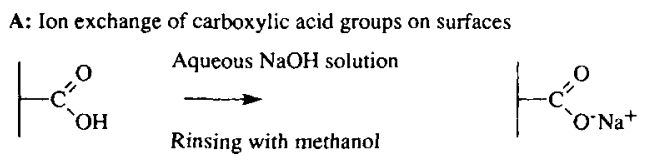

B: Reaction with trifluoroethanol

Pyridine $(\mathrm{g})$, di-tertbutylcarbodimide $(\mathrm{g})$

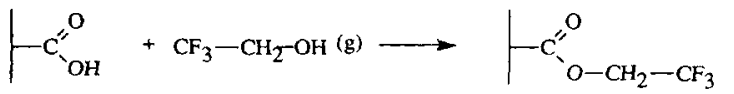

Scheme 1 Derivatization reactions used to tag carboxylic acid groups on surfaces. The modified surfaces are analyzed with XPS.
Ion Exchange of Carboxylic Acid Groups. By rinsing a carboxylic acid group containing surface with an aqueous $\mathrm{NaOH}$ solution and by quantification of the amount of sodium on the surface by XPS, it is possible to obtain an indication of the surface concentration of carboxylic acid groups. ${ }^{26}$ Because the rinsing procedure, which has to be applied to remove an excess of $\mathrm{NaOH}$ from the surface, is critical, the washing procedure had to be optimized. Therefore a model polymer surface, $\mathrm{P}$ (EcoAAc), was treated with aqueous $\mathrm{NaOH}(1.00 M, 1 \mathrm{~h}, \mathrm{RT})$, rinsed varying times with methanol, and dried in vacuo. After rinsing twice with methanol a constant sodium level (half of the oxygen concentration) was found on the surface with XPS. The ion-exchange of surface carboxylic acid groups was thus complete, and the sodium concentration could be used to quantify the surface concentration of carboxylic acid groups. This procedure has been used for all plasma-treated surfaces.

Reaction of Carboxylic Acid Groups With TFE. By reacting surface carboxylic acid groups with TFE and quantification of the F1s peak during XPS analysis, it is possible to get a (semi)quantitative indication of the carboxylic acid concentration on the surface. This reaction has been described by Chilkoti et al. ${ }^{26}$ A slight modification of the method described was used. A polymer film $(13 \times 25 \mathrm{~mm})$ was placed vertically in a glass vial on glass beads. Subsequently TFE $(0.45 \mathrm{~mL})$, pyridine $(0.2 \mathrm{~mL})$, and 1,3-di-tert-butylcarbodiimide $(0.15 \mathrm{~mL})$ were injected under the film at 15-min intervals. The vial was closed and the reaction was allowed to proceed for $24 \mathrm{~h}$ at $\mathrm{RT}$. After the reaction the film was immediately loaded for XPS analysis. The semiquantitative nature of this method was checked by reacting PAAc films according to this procedure. XPS analysis of the derivatized surfaces showed that about $80 \%$ of the carboxylic acid groups on the surface have been derivatized, which is in close agreement with the results of Chilkoti et al. ${ }^{26}$

\section{RESULTS AND DISCUSSION}

\section{Adsorption of PAAc on PE and Desorption of PAAC From PE}

The adsorption of PAAc on PE and the desorption of PAAc from PE have been studied with XPS and water contact angle measurements ( see Table I). It can be seen from Table I that PAAc adsorbs on PE from aqueous PAAc solutions. PE/PAAc films con- 
Table I Adsorption of PAAc on PE and Desorption of PAAc From PE

\begin{tabular}{lcccr} 
& \multicolumn{4}{c}{ Atom Percentages } \\
\cline { 2 - 5 } \multicolumn{1}{c}{ Sample } & $\mathrm{O}$ & $\mathrm{F}$ & $\mathrm{Na}$ & $\Theta_{\mathrm{adv}}$ \\
\hline PE & $0.3 \pm 0.5$ & $0.1 \pm 0.1$ & & $111 \pm 3$ \\
PE/PAAc & $3.3 \pm 0.7$ & $0.1 \pm 0.1$ & & $102 \pm 1$ \\
PE + TFE & 0.3 & 0.1 & & $78 \pm 2$ \\
PE/PAAc + TFE & 4.8 & 4.7 & $0.1 \pm 0.2$ & $114 \pm 3$ \\
PE washed & $0.2 \pm 0.1$ & - & $0.1 \pm 0.1$ & $113 \pm 3$ \\
PE/PAAc washed & $0.4 \pm 0.3$ & - & & $88 \pm 3$ \\
PE washed, + TFE & 0.4 & 0.1 & & $88 \pm 4$ \\
PE/PAAc washed, + TFE & 0.3 & 0.2 & & \\
\hline
\end{tabular}

The results of XPS and contact angle measurements before and after washing with an aqueous NaOH solution for PE and for PE preadsorbed with PAAc (PE/PAAc) are given. The results of the XPS analysis of TFE derivatized PE and PE/PAAc films before and after washing are also shown. The average values of two measurements are presented. If a standard deviation is given, the average of four measurements is denoted.

tain a significantly higher oxygen level on the surface as compared to the PE control surfaces. Derivatization of PE/PAAc films with TFE followed by XPS analysis shows that the increase of the oxygen concentration of the PE/PAAc films is due to an increased amount of carboxylic acid groups (Table I). The surface concentration of fluorine of the TFE derivatized PE/PAAc surface is slightly lower than the expected value $[5.8 \%(=4.8 \times 3 / 2 \times 0.80)$ fluorine] based on the amount of oxygen present on the surface and the reaction efficiency. It seems likely that the adsorption of PAAc on PE is caused by hydrophobic interactions of the PAAc backbone with the PE substrate.

The presence of PAAc on the PE surface after adsorption is also confirmed by contact angle measurements (Table I). Adsorption of a hydrophilic polymer onto a hydrophobic surface should cause a decrease of the advancing and receding contact angles, which is also observed. However, it should be noted that measuring contact angles of PE/PAAc films, which are not stable in water, may lead to erroneous results and that the data should be interpreted with great caution.

In order to study the effect of the PAAc solution concentration on the amount of PAAc adsorbed on $\mathrm{PE}$, the PAAc solution concentrations were varied from 0.25 up to $2.5 \% \mathrm{w} / \mathrm{v}$. It was found with XPS measurements that the amount of PAAc adsorbed on the surface does not depend on the concentration of PAAc used for the adsorption process within this concentration range (data not shown).

By washing the PE/PAAc surfaces with an aqueous $\mathrm{NaOH}$ solution, the surface properties of the films became comparable to similarly washed
PE and unwashed PE films. The amount of oxygen on the surface decreased sharply and the contact angles became higher (Table I). Furthermore, derivatization of washed surfaces with TFE showed that no carboxylic acid groups are present on the surface after washing. The preadsorbed PAAc can be efficiently removed from the surface by washing PE/PAAc surfaces with an aqueous $\mathrm{NaOH}$ solution.

Similar results were obtained when PE/PAAc films were washed with either $3.0 \mathrm{M} \mathrm{NaCl}$ or $1 \% \mathrm{w} /$ $\mathrm{v}$ SDS. The surface properties of PE/PAAc films washed with $1 \mathrm{M} \mathrm{HCl}$ were similar to those of unwashed PE/PAAc films (data not shown).

Because washing with $\mathrm{NaOH}(1 M)$ and rinsing twice with methanol is a convenient way to detect surface-bound carboxylic acid groups, and because a preadsorbed PAAc layer on PE can also be removed by this washing procedure, this method was used to detect carboxylic acid groups on surfaces of plasma-treated PE/PAAc and PE films.

\section{Argon Plasma Treatment of PE/PAAc and PE Films}

From Figure 3 in which the results of the XPS analysis of argon plasma-treated PE/PAAc and PE films are given, it can be seen that after washing with an aqueous $\mathrm{NaOH}$ solution no clear differences between $\mathrm{PE} / \mathrm{PAAc}$ and PE films were found. The amount of oxygen on PE and PE/PAAc films after washing increased with increasing plasma treatment times. A constant, low sodium level was also found, which is an indication that a small amount of bound carboxylic acid groups is present on both surfaces. From 

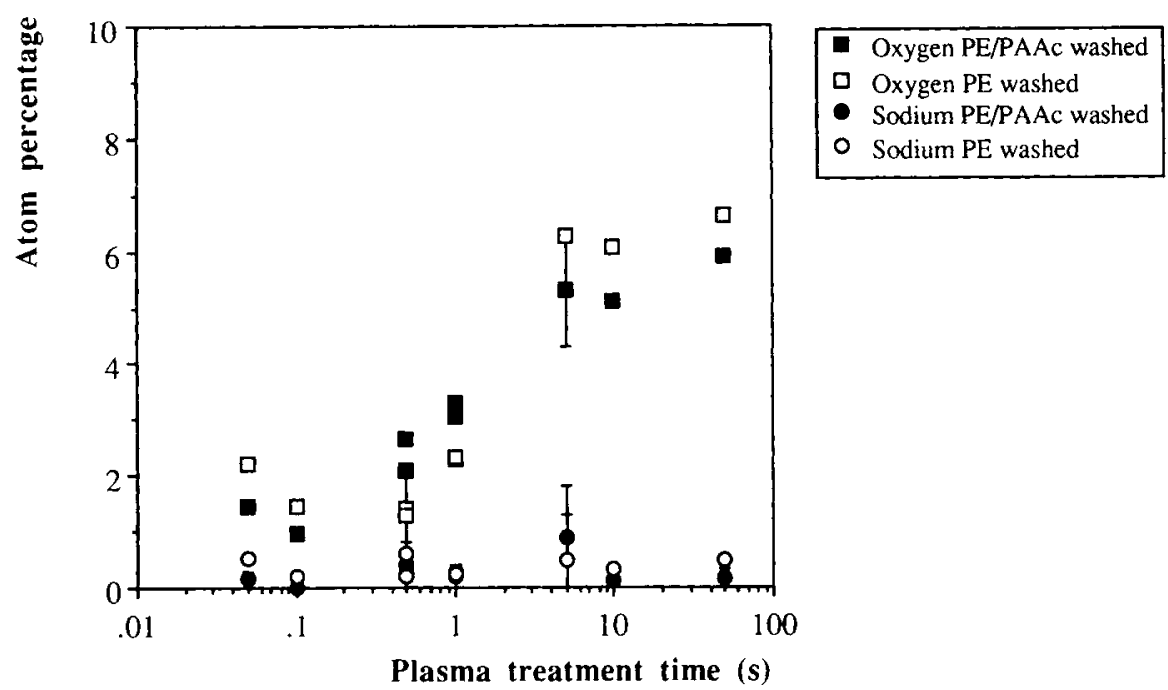

Plasma treatment time (s)

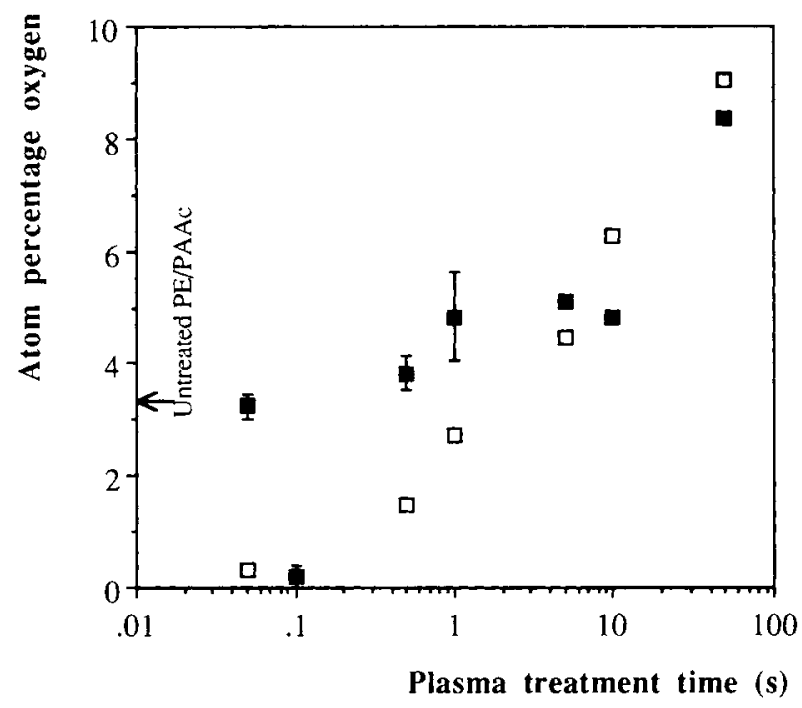

DE not washed

- PE/PAAc not washed

Figure 3 XPS analysis of argon plasma-treated PE/PAAc and PE films. The films were either (a) washed with an aqueous $\mathrm{NaOH}$ solution to remove any unbound PAAc and to tag carboxylic acid groups on the surface, or (b) not washed after the plasma treatment. The atom percentages of oxygen and sodium are given as a function of the plasma treatment time. The error bars when given represent the standard deviation of the results obtained for three films within one batch.

the contact angles for washed PE/PAAc and PE films given in Figure 4, it can be seen that no differences between plasma-treated PE/PAAc and PE films after washing can be found. It can thus be concluded that an argon plasma treatment applied on a layer of PAAc preadsorbed on PE does not result in an increase of the amount of carboxylic acid groups on the surface.

To investigate why the immobilization of the preadsorbed PAAc layer on PE failed, plasma- treated PE/PAAc and PE films, which were not washed afterward, were analyzed with XPS. For unwashed PE/PAAc films the amount of oxygen initially decreases and then increases at longer plasma treatment times [Fig. 3(b)]. This is in contrast to the plasma-treated PE films for which the amount of oxygen on the surface increases monotonously.

The data given in Figure 3(b) show that after a plasma treatment time of $0.05 \mathrm{~s}$ a significant amount of oxygen is observed on the surface, but that after 


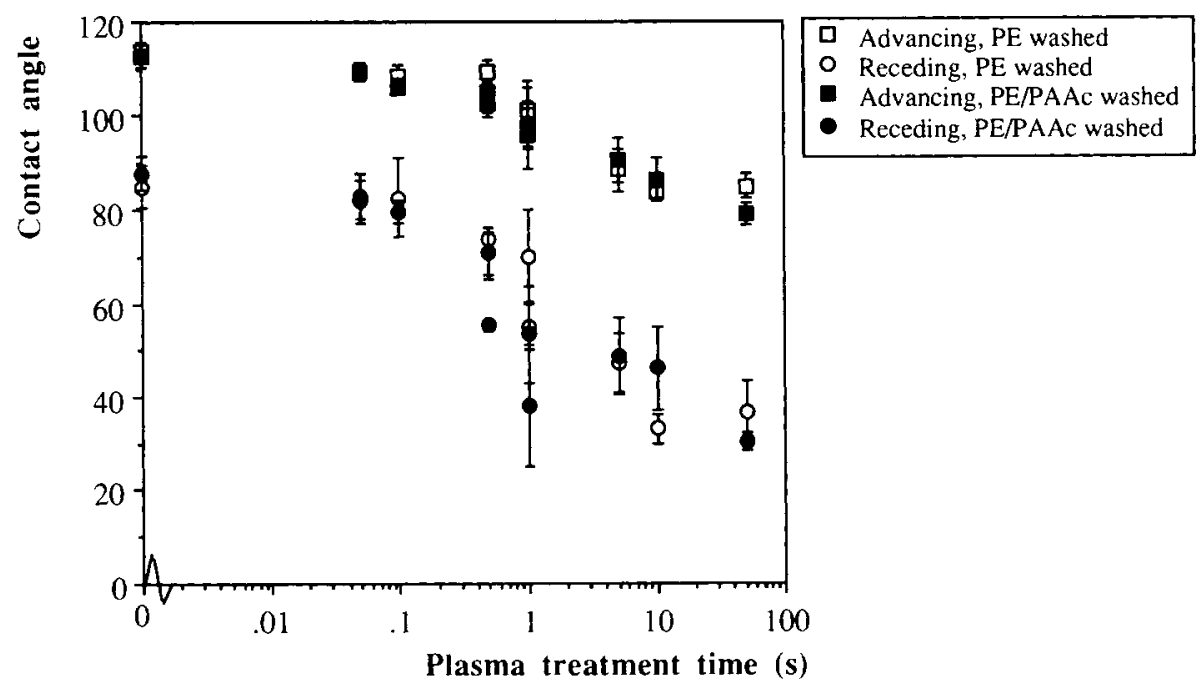

Figure 4 Water contact angle data of argon plasma-treated and washed (with an aqueous $\mathrm{NaOH}$ solution to remove any unbound PAAc) PE and PE/PAAc films. The advancing and receding water contact angles are given as functions of the plasma treatment time. The error bars represent the standard deviation in the contact angles of three films within one batch.

$0.1 \mathrm{~s}$ the preadsorbed layer is etched off from the $\mathrm{PE}$ surface. However the treatment time of $0.05 \mathrm{~s}$ was not sufficient to couple the preadsorbed PAAc to the PE substrate [Fig. 3(a)]. Compared to the immobilization of decylamine hydrochloride on $\mathrm{PE}$ for which under almost identical plasma conditions an optimal plasma treatment time of $2 \mathrm{~s}$ has been observed, ${ }^{22}$ these treatment times are extremely short. The etching rates given in Table II illustrate the sensitivity of PAAc to argon plasmas. Because the immobilization of a layer preadsorbed on a hydrophobic surface by a plasma involves a competition between an etching and an immobilization process, ${ }^{21}$ it seems likely that when the etching process is very prominent, hardly any immobilization will occur. In the case of the immobilization of PAAc on

Table II Gravimetrically Determined Etching Rates of PE and PAAc Films During Different Plasma Treatments

\begin{tabular}{lrrr}
$\begin{array}{c}\text { Etching Rate } \\
(\AA / \mathrm{min})\end{array}$ & Argon & $\mathrm{CF}_{4}$ & Air \\
\hline PE & 6 & 70 & 600 \\
PAAc & 200 & 200 & 1200 \\
\hline
\end{tabular}

The densities used to convert the weight decrease into an etching rate are: PE $0.93 \mathrm{~g} / \mathrm{cm}^{3}$ and PAAc $1.3 \mathrm{~g} / \mathrm{cm}^{3}$.
PE by an argon plasma, the etching process seems to be too dominant.

Although the only difference in chemical structure between PAAc and PE is the pendant carboxylic acid group along the backbone, the etching rates during different plasma treatments differ greatly. PE is etched slowly, probably due to a sputtering process of the surface. This is in contrast to the high etching rates observed for PAAc. For PAAc an additional process, presumably decarboxylation, occurs during the plasma treatment. The exact chemical modifications of PAAc induced by an argon plasma are presently under investigation.

Generally the surface concentration of oxygen on argon plasma-treated films increases with increasing plasma treatment time. The argon plasma introduces active sites on a polymer surface by a bombardment of reactive species (ions, electrons, and $\mathrm{UV}^{13}$ ) from the plasma phase. These active sites, presumably radicals, can either recombine to give crosslinking, ${ }^{30,31}$ or can cause immobilization of a preadsorbed compound to the substrate..$^{21,22}$ In addition these active sites can also react with oxygencontaining compounds like water adsorbed on the reactor walls or air from leakages. Oxidation may also occur after the plasma treatment. ${ }^{32}$ These reactions will yield different oxygen-containing functionalities on the surface including a small amount of carboxylic acid groups. 
A systematic study on the effect of treating polymer films with a plasma for very short time periods has not been reported previously. From the XPS data given in Figure $3(\mathrm{~b})$ it can be derived that the kinetics of treating a PE film with an argon plasma can be divided into three regions. First, up to treatment times of $0.1 \mathrm{~s}$ the argon plasma does not induce changes in the surface composition of PE films. After this induction period, the incorporation of oxygencontaining groups seems to be proportional to the
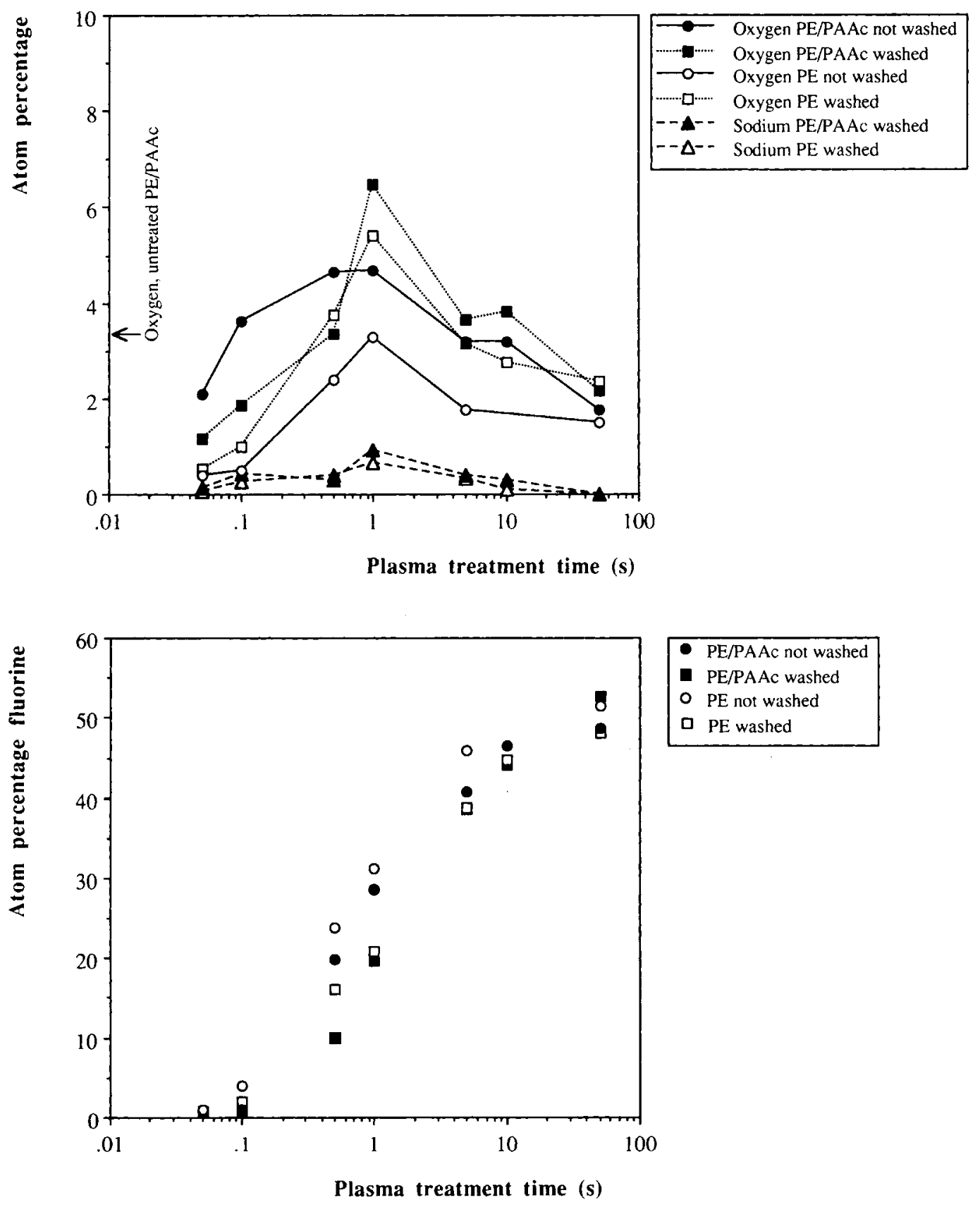

Figure 5 XPS analysis of $\mathrm{CF}_{4}$ plasma-treated PE/PAAc and PE films. The films were either washed with an aqueous $\mathrm{NaOH}$ solution to remove any unbound PAAc and to tag carboxylic acid groups on the surface, or not washed after the plasma treatment. The atom percentages of (a) oxygen and sodium and (b) fluorine are given as a function of the plasma treatment time. 
log of the plasma treatment time (second region). It can be expected that at long treatment times ( $t$ $>50 \mathrm{~s}$ ) the surface oxidation and the etching of the surface are each occurring at fixed rates leading to a steady state (third region). This will yield a constant surface composition.

\section{$\mathrm{CF}_{4}$ Plasma Treatment of PE/PAAC and PE Films}

Because the immobilization of PAAc on PE by an argon plasma treatment was not successful, a different type of plasma treatment was tried. We expected that plasmas of other noble gases would give similar results and therefore a totally different type of gas $\left(\mathrm{CF}_{4}\right)$ was utilized. PE/PAAc and PE films (as control surfaces) have thus been treated with $\mathrm{CF}_{4}$ plasma. The results of the XPS analysis of plasma-treated and washed films, given in Figure 5, show that no differences between PE and PE/PAAc films are found. The PAAc layer preadsorbed on PE can therefore also not be immobilized by a $\mathrm{CF}_{4}$ plasma treatment.

The maximum in the oxygen content of $\mathrm{CF}_{4}$ plasma-treated PE films is remarkable. This maximum is most likely due to the consumption of a limited internal oxygen source in the reactor (e.g., adsorbed water/oxygen) during the plasma treatment. Part of the incorporated oxygen is present as carboxylic acid groups.

This conclusion is supported by the water contact angle measurements given in Figure 6. Although small differences between PE/PAAc and PE films are found at plasma treatment times of $0.5,1$, and $5 \mathrm{~s}$, these seem to be more related to small differences in the fluorine and oxygen content of these surfaces than to differences in the amount of carboxylic acid groups on the surface. The maximum in the amount of oxygen on the surface is related to the minimum in the contact angles of Figure 6.

When the oxygen levels of $\mathrm{CF}_{4}$ plasma-treated PE/PAAc and PE films are compared before washing, a significantly higher amount of oxygen is found on the PE/PAAc films [Fig. 5(a)]. Only at a plasma treatment time of $50 \mathrm{~s}$ the amounts of oxygen on both surfaces become comparable. The initially higher amount of oxygen on PE/PAAc films is most likely due to the preadsorbed PAAc layer. This layer is removed during the washing procedure.

The $\mathrm{CF}_{4}$ plasma treatment also results in the incorporation of large amounts of fluorine on the surface [Fig. $5(\mathrm{~b})$ ]. It has been reported that a $\mathrm{CF}_{4}$ plasma treatment of a polymer surface leads to the

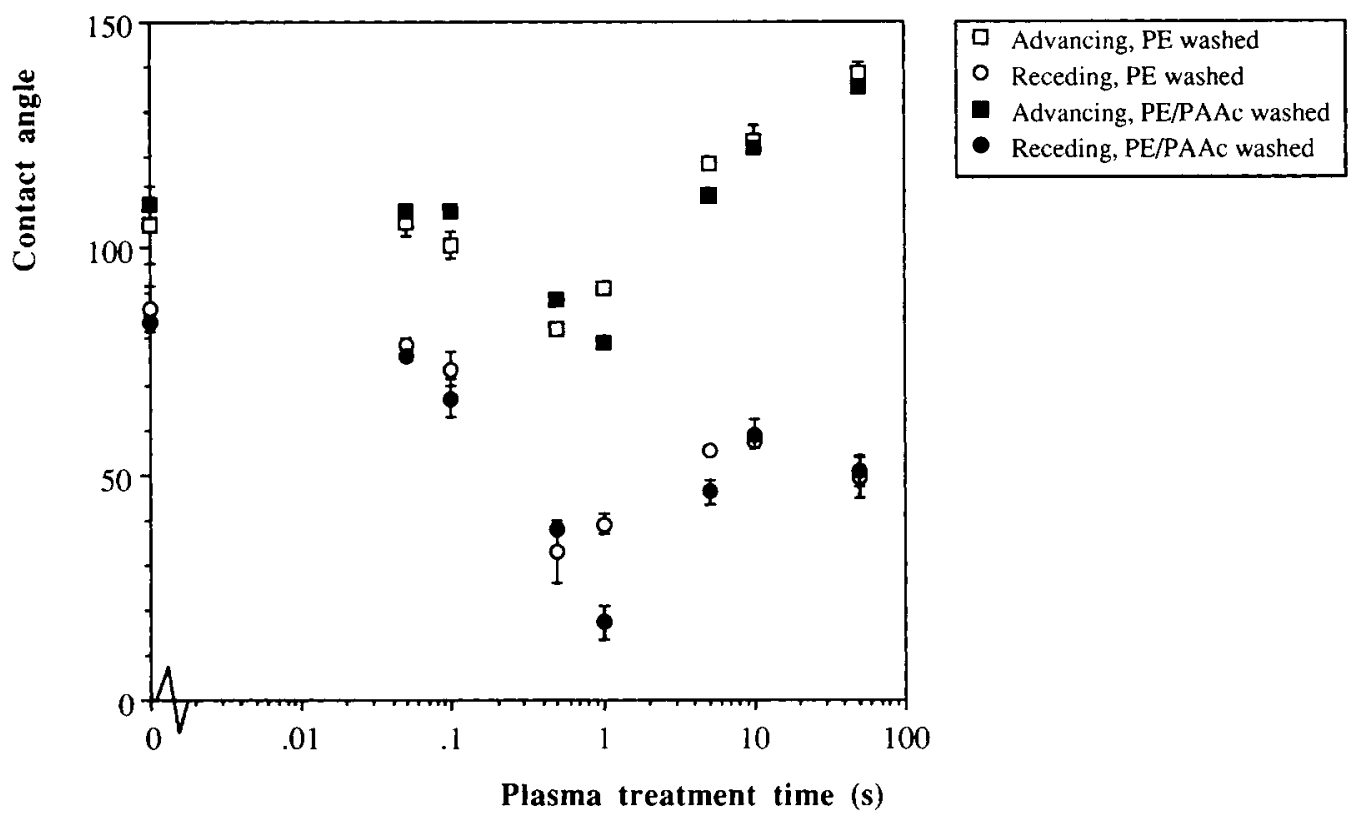

Figure 6 Water contact angles of $\mathrm{CF}_{4}$ plasma-treated and washed (with an aqueous $\mathrm{NaOH}$ solution to remove any unbound PAAc) PE and PE/PAAc films. The advancing and receding water contact angles are given as a function of the plasma treatment time. The error bars represent the standard deviation in the contact angles of three films within one batch. 
formation of $\mathrm{CF}, \mathrm{CF}_{2}$, and $\mathrm{CF}_{3}$ groups. ${ }^{33,34}$ The $\mathrm{C1s}$ spectra of $\mathrm{CF}_{4}$ plasma-treated $\mathrm{PE}$ films showed that indeed $\mathrm{CF}, \mathrm{CF}_{2}$, and $\mathrm{CF}_{3}$ groups are incorporated at the surface ( spectra not shown). Furthermore these spectra showed that at short plasma treatment times predominantly $\mathrm{CF}$ groups are built in and at longer plasma treatment times an increasing amount of $\mathrm{CF}_{2}$ and small amounts of $\mathrm{CF}_{3}$ groups are formed. These observations may be explained by the progressive fluorination of the surface by atomic fluorine. ${ }^{20}$

From Table II it can be seen that PAAc films are also sensitive to a $\mathrm{CF}_{4}$ plasma treatment. Although $\mathrm{PE}$ films are also more rapidly etched by $\mathrm{CF}_{4}$ than by argon plasmas, the etching rate of PAAc films is still significantly higher. It seems that again a second process, possibly decarboxylation, occurs during the $\mathrm{CF}_{4}$ plasma treatment of PAAc. This would explain the failure of the covalent immobilization of a layer of PAAc preadsorbed on $\mathrm{PE}$ by a $\mathrm{CF}_{4}$ plasma.

For the treatment of $\mathrm{PE}$ with a $\mathrm{CF}_{4}$ plasma, some kinetic aspects can be derived from Figure 5(b). The classification of the kinetics into three regions, as was described for the argon plasma treatment of $\mathrm{PE}$, can also be applied to the fluorination of the $\mathrm{PE}$ surface during a $\mathrm{CF}_{4}$ plasma. Again after a short induction period $(0.1 \mathrm{~s})$, the fluorine incorporation is proportional to the log of the treatment time. In contrast to the argon plasma treatment the onset of the third region, in which the incorporation and etching process are balancing, can be seen in Figure 5 (b) at a plasma treatment time of $50 \mathrm{~s}$.

\section{CONCLUSIONS}

In this study a layer of PAAc reversibly adsorbed on $\mathrm{PE}$ was treated with either an argon or a $\mathrm{CF}_{4}$ plasma in order to immobilize a preadsorbed PAAc layer to the PE substrate. It was not possible to introduce an additional amount of carboxylic acid groups on the surface by this method compared to PE control surfaces. The preadsorbed PAAc layer on $\mathrm{PE}$ is etched off before it could be immobilized by either an argon or a $\mathrm{CF}_{4}$ plasma treatment. Additionally the effect of treating $\mathrm{PE}$ films for very short times with an argon or $\mathrm{a} \mathrm{CF}_{4}$ plasma was studied. After an induction period of approximately 0.1 $\mathrm{s}$, the oxidation during the argon plasma treatment and the fluorination during the $\mathrm{CF}_{4}$ plasma treatment, were proportional to the logarithmic of the treatment time for time periods up to $50 \mathrm{~s}$.

The authors would like to thank Dr. G. Engbers for his valuable contributions during discussions, Mr. L. Hassink his technical advice on the plasma apparatus, and Mr. A. van den Berg for assisting with the XPS measurements. Furthermore, they are grateful to DSM (Geleen, The Netherlands) for financial support for this research.

\section{REFERENCES}

1. Y. Inaki, in Functional Monomers and Polymers, Procedures, Synthesis, Applications, Chap. 9, K. Takemoto, Y. Inaki, and R. M. Ottenbrite, Eds., Marcel Dekker, Inc., New York, 1987.

2. D. Briggs, D. M. Brewis, and M. B. Konieczo, J. Mater. Sci., 11, 1270-1277 (1976).

3. S. R. Holmes-Farley and G. M. Whitesides, Langmuir, 3, 62-76 (1987).

4. S. R. Holmes-Farley, R. H. Reamey, R. Nuzzo, T. J. McCarthy, and G. M. Whitesides, Langmuir, 3, 799 815 (1987).

5. S. R. Holmes-Farley, C. D. Bain, and G. M. Whitesides, Langmuir, 4, 921-937 (1988).

6. Y. Avny, L. Rebenfeld, and H.-D. Weigmann, J. Appl. Polym. Sci., 22, 125-147 (1978).

7. I. K. Mehta, S. Kumar, G. S. Chauhan, and B. N. Misra, J. Appl. Polym. Sci., 41, 1171-1180 (1990).

8. G.-H. Hsiue and C.-C. Wang, J. Appl. Polym. Sci., 40, 235-247 (1990).

9. I. Ishigaki, T. Sugo, K. Senoo, T. Okada, J. Okamoto, and S. Machi, J. Appl. Polym. Sci., 27, 1033-1041 (1982).

10. I. Ishigaki, T. Sugo, T. Takayama, T. Okada, J. Okamoto, and S. Machi, J. Appl. Polym. Sci., 27, 10431051 (1982).

11. Z. P. Yao and B. Rånby, J. Appl. Polym. Sci., 40, 1647-1661 (1990).

12. S. Wu, Polymer Interface and Adhesion, Marcel Dekker, New York, 1982.

13. H. Yasuda, J. Macromol. Sci.-Chem., A10, 383-420 (1976).

14. G. H. Heider, M. B. Gelbert, and A. M. Yacynych, Anal. Chem., 54, 322-324 (1982).

15. D. L. Cho, P. M. Claesson, C.-G. Gölander, and K. Johansson, J. Appl. Polym. Sci, 41, 1373-1390 (1990).

16. N. Inagaki and M. Matsunaga, Polym. Bull., 13, 349352 (1985).

17. J. Meischer and H.-U. Poll, Acta Polymerica, 32, 203208 (1981).

18. A. Bradley and J. D. Fales, Chem. Tech April, 232237 (1971).

19. H. Iwata and T. Matsuda, J. Membrane Sci., 38, 185 199 (1988).

20. F. D. Egitto, V. Vukanovic, and G. N. Taylor, in Plasma Deposition, Treatment, and Etching of Polymers, Chapt. 5, R. d'Agostino, Ed., Academic Press, Boston, 1990.

21. J. G. A. Terlingen, J. Feijen, and A. S. Hoffman, J. Colloid Interface Sci., 155, 55-65 (1993). 
22. J. G. A. Terlingen, L. M. Brenneisen, H. T. J. Supèr, A. P. Pijpers, A. S. Hoffman, and J. Feijen, J. Biomater. Sci. Polym. Edn., 4, 165-181 (1993).

23. M.-S. Sheu, A. S. Hoffman, J. G. A. Terlingen, and J. Feijen, J. Clin. Mater., to appear.

24. M.-S. Sheu, A. S. Hoffman, and J. Feijen, J. Adhesion Sci. Technol., 6, 995-1009 (1992).

25. A. M. Rothman, European Pat., EP 0135298 A2 (1985).

26. A. Chilkoti, B. D. Ratner, and D. Briggs, Chem. Mater., 3, 51-61 (1991).

27. J. Andrade, in Surface and Interfacial Aspects of Biomedical Polymers, Chapt. 5, J. Andrade, Ed., Plenum Press, New York, 1985.

28. H. van Damme, A. H. Hogt, and J. Feijen, J. Colloid Interface Sci., 114, 167-172 (1986).

29. H. S. van Damme, A. H. Hogt, and J. Feijen, in Poly- mer Surface Dynamics, J. D. Andrade, Ed., Plenum Press, New York, 1988, pp. 89 100.

30. R. H. Hansen and H. Schonhorn, J. Polym. Sci., Polym. Lett., 4, 203-209 (1966).

31. D. T. Clark and A. Dilks, J. Polym. Sci., Polym. Chem. Ed., 15, 2321-2345 (1977).

32. L. J. Gerenser, J. Adhesion Sci. Tech., 1, 303-318 (1987).

33. A. Bruil, L. M. Brenneisen, J. G. A. Terlingen, T. Beugeling, W. G. van Aken, and J. Feijen, J. Colloid Interface Sci., to appear.

34. T. Yasuda, T. Okuno, K. Yoshida, and H. Yasuda, $J$. Appl. Polym. Sci. B, Polym. Phys., 26, 1781-1794 (1988).

Received March 11, 1993

Accepted April 2, 1993 